\title{
Estudo Randomizado e Comparativo da Intervenção Coronária Percutânea com Stents Recobertos por Titânio-Óxido Nítrico ou de Aço Inoxidável em Pacientes com Doença Arterial Coronária: Estudo RIO
}

\author{
Fernando Mendes Sant'Anna', Leonardo Alves Batista ${ }^{1}$, Marcelo Bastos Brito', Sérgio Menezes', \\ Fábio Machado Ventura ${ }^{1}$, Leonardo Buczynski ${ }^{1}$, Carlos Alberto Mussel Barrozo
}

\section{RESUMO}

Introdução: $O$ stent recoberto por titânio-óxido nítrico (Titan) mostrou-se eficaz para redução da hiperplasia neointimal comparado ao stent convencional em animais e seres humanos. Foi elaborado um estudo clínico prospectivo e randomizado do stent Titan versus stent de aço inoxidável, cujo objetivo foi comparar o índice de eventos cardíacos adversos maiores (ECAM) após dois anos. Método: No período de outubro de 2005 a maio de 2006, 200 pacientes (228 lesões) foram submetidos a implante de stent eletivo e randomizados para dois grupos: I) stent metálico (100) e II) stent Titan (100). O acompanhamento dos doentes foi realizado por consulta clínica. ECAM foram definidos como: morte, infarto agudo do miocárdio ou nova revascularização da lesão-alvo. As sobrevidas livres de ECAM e angina após dois anos foram comparadas entre os dois grupos. Resultados: Foi obtido sucesso em todos os procedimentos. As características clínicas e angiográficas foram similares nos dois grupos. O período médio de seguimento foi de $482 \pm 264$ dias. Aos 30 dias, o índice de ECAM foi de $2 \%$ no grupo I e de $1 \%$ no grupo II ( $P=0,32$ ). Após dois anos, a sobrevida livre de ECAM foi melhor no grupo Titan que no grupo stent metálico (93,6\% vs. 82,4\%; $\mathrm{P}=0,036)$, assim como a sobrevida livre de angina $(84 \%$ vs. $71 \% ; P=0,02)$. Conclusões: $O$ implante do stent Titan em pacientes com insuficiência coronariana crônica é seguro e efetivo, e cursa com índices de ECAM e angina após dois anos menores que os do stent de aço inoxidável.

DESCRITORES: Contenedores. Stents farmacológicos. Reestenose coronária.

\section{ABSTRACT}

Randomized Comparison of Percutaneous Coronary Intervention with Titanium-Nitride-OxideCoated Stents Versus Stainless Steel Stents in Patients with Coronary Artery Disease: RIO Trial

Background: Stent coating with titanium-nitride oxide (Titan) has been shown to reduce neointimal hyperplasia in both animals and humans. A prospective, randomized, clinical study was designed to compare the incidence of major adverse cardiac events (MACE) after two years in patients with the Titan stent versus the stainless steel stent. Methods: Two hundred patients (228 stenosis) submitted to stent implantation between October 2005 and May 2006 were randomized into two groups: I) Bare metal stent (100); II) Titan (100) and followed clinically. MACE was defined as a composite of death, acute myocardial infarction or clinically driven revascularization of target lesion. MACE and angina free survival after 2 years were compared between the two groups. Results: All procedures were successful in both groups. Baseline clinical and angiographic characteristics were similar in both groups. Mean followup period was $482 \pm 264$ days. After 30 days, MACE was $2 \%$ in group I and $1 \%$ in group $I I(P=0.32)$. MACE free survival at 2 years was significantly better in the Titan group than in the bare metal stent group $(93.6 \%$ vs. $82.4 \%$; $P=0.036)$ as well as angina free survival $(84 \%$ vs. $71 \%$; $P=0.02)$. Conclusions: Revascularization with Titan stent in chronic coronary artery disease is safe, effective, and reduces the incidence of MACE and angina after two years when compared with the bare metal stent.

DESCRIPTORS: Stents. Drug-eluting stents. Coronary restenosis.

1 Hospital Santa Helena - Cabo Frio, RJ, Brasil.

Correspondência: Fernando Mendes Sant'Anna. Rua Safira, 20 -

Portinho - Cabo Frio, RJ, Brasil - CEP 28915-400

E-mail: fmsantanna@bol.com.br

Recebido em: 22/2/2009 • Aceito em: 20/3/2009 

Nítrico ou de Aço Inoxidável em Pacientes com Doença Arterial Coronária: Estudo RIO. Rev Bras Cardiol Invas. 2009;17(1):69-75.

A reestenose permanece ainda como o calcanhar de Aquiles da angioplastia1,2. Os stents de aço inoxidável eliminaram o problema do remodelamento arterial e da retração elástica do vaso, mas a hiperplasia neointimal decorrente do implante desse tipo de stent é responsável por índice de reestenose em torno de $30 \%{ }^{3}$. Os stents farmacológicos diminuíram substancialmente a reestenose ${ }^{4}$, porém o custo elevado limita sua utilização a apenas uma pequena parcela dos pacientes atendidos em nosso País.

Modificações na geometria do stent ${ }^{5}$, na espessura das hastes ${ }^{6}$ e na cobertura de sua superfície ${ }^{7}$ também têm se mostrado úteis na redução da reestenose intrastent, porém sem atingir resultados satisfatórios. Recentemente, demonstrou-se que pacientes com história de alergia ao níquel apresentavam taxas de reestenose elevadas após implante de stents metálicos ${ }^{8}$. O níquel é um componente importante do aço inoxidável, o que sugere que o material de que é feito o stent tem papel importante nos mecanismos de reparo vascular após a injúria arterial provocada pelo implante da prótese.

O titânio, um material frequentemente utilizado em próteses biológicas, apresenta biocompatibilidade superior ao ouro, ao aço inoxidável e a outros produtos utilizados na superfície dos stents ${ }^{9}$. Ligado ao óxido nítrico, o titânio pode ser facilmente depositado por meio de vaporização na superfície dos stents de aço inoxidável. Estudos in vitro demonstraram que stents recobertos por titânio-óxido nítrico (Titan) apresentavam diminuição da adesão plaquetária e ligação de fibrinogênio, quando comparados aos stents convencionais de aço inoxidável ${ }^{10}$. Um estudo em animais demonstrou redução importante da hiperplasia neointimal em porcos que receberam stent recoberto por titânioóxido nítrico em relação ao stent de aço inoxidável, num período de seguimento de 6 semanas $^{11}$.

Em seres humanos, diversos estudos publicados recentemente demonstraram índices reduzidos de reestenose e eventos cardíacos adversos maiores após seis meses a um ano em pacientes submetidos a implante de stent recoberto por titânio-óxido nítrico (entre $7 \%$ e $11 \%)^{12-14}$. No único estudo randomizado no qual o stent Titan foi comparado aos stents de aço inoxidável, a redução foi bem mais significativa $(7 \%$ vs. $27 \%$; $P=0,02)^{12}$. Num estudo prospectivo, observacional, no qual o Titan foi comparado ao stent recoberto por paclitaxel, o índice de eventos cardíacos adversos maiores após um ano foi semelhante nos dois grupos $(10,9 \% \text { vs. } 13,7 \% ; P=0,40)^{15}$.

O objetivo do Estudo Randomizado e Comparativo da Intervenção Coronária Percutânea com Stents Recobertos por Titânio-Óxido Nítrico ou de Aço Inoxidável em Pacientes com Doença Arterial Coronária (RIO), prospectivo, foi comparar, em pacientes submetidos a implante de stent eletivo num período consecutivo de tempo, o índice de eventos cardiovasculares adversos maiores após dois anos em dois grupos de pacientes, um deles recebendo stent recoberto por titânio-óxido nítrico (stent Titan, Hexacath - Rueil-Malmaison, França) e o outro, stent convencional de aço inoxidável.

\section{MÉTODO}

A casuística desse estudo constituiu-se de 200 pacientes encaminhados para nosso Serviço, no período de outubro de 2005 a maio de 2006, para angioplastia coronariana eletiva. Os critérios de inclusão foram: 1) presença de sintomas ou sinais objetivos de isquemia miocárdica; e 2) lesão maior que 50\% (pela estimativa visual) em artéria coronária principal (direita, circunflexa ou descendente anterior) ou ramo de grande importância. Como critérios de exclusão, foram adotados: 1) infarto agudo do miocárdio; 2) lesão igual ou maior que $50 \%$ no tronco da artéria coronária esquerda; 3) história de alergia ou contraindicação ao uso de ácido acetilsalicílico (AAS) ou clopidogrel e/ou ticlopidina; 4) paciente com menos de 21 anos de idade; 5) gravidez; e 6) recusa em assinar o termo de consentimento. É importante mencionar que pacientes com angina instável só foram incluídos no estudo depois de estabilizados clinicamente e tratados eletivamente, conforme conduta adotada em nosso Serviço.

O tamanho da amostra de nosso estudo foi determinado partindo-se da seguinte premissa: de acordo com dados da literatura, o índice de eventos cardíacos adversos maiores em pacientes submetidos a implante de stents convencionais de aço inoxidável varia entre $22 \%$ e $27 \%$, enquanto em pacientes submetidos a implante de stent recoberto por titânio-óxido nítrico esse índice varia entre $6 \%$ e $10 \%{ }^{12-14}$. Considerandose a incidência de eventos cardíacos adversos maior que $7 \%$ para o grupo Titan e de $25 \%$ para o grupo stent convencional, para um poder do teste de 0,8 com nível de significância de 5\%, seriam necessários 64 pacientes em cada grupo (total: 128). Para trabaIharmos com boa margem de segurança, resolvemos incluir no estudo 200 pacientes.

Antes da intervenção, os pacientes foram randomizados, por meio de números sequenciais gerados aleatoriamente por computador (com emprego do software Stata SE), para receber stent metálico de aço inoxidável (grupo stent convencional) ou stent recoberto por titânio-óxido nítrico (grupo Titan). Cada número aleatório correspondia a um grupo. Esses números/ grupos foram colocados dentro de envelopes opacos e foram lacrados (colados), no próprio centro onde se desenvolveu o estudo, por uma secretária que não tinha conhecimento do propósito do estudo. Os envelopes, abertos sequencialmente à medida que os pacientes eram recrutados para o estudo (de forma consecutiva), continham o grupo ao qual o paciente pertenceria.

O objetivo primário do estudo era determinar o índice de eventos cardíacos adversos maiores nos dois 
Sant'Anna FM, et al. Estudo Randomizado e Comparativo da Intervenção Coronária Percutânea com Stents Recobertos por Titânio-Óxido Nítrico ou de Aço Inoxidável em Pacientes com Doença Arterial Coronária: Estudo RIO. Rev Bras Cardiol Invas. 2009;17(1):69-75.

grupos após dois anos. Como eventos cardíacos adversos maiores definiu-se o composto de morte, infarto agudo do miocárdio ou nova revascularização da lesão-alvo. O objetivo secundário foi determinar a quantidade de pacientes livres de angina nesse mesmo período. Definiu-se como infarto agudo do miocárdio a elevação dos níveis de creatina quinase fração $M B(C K-M B)$ após o procedimento maior que 2,5 vezes o valor basal, com ou sem alteração de eletrocardiograma. Como revascularização da lesão-alvo definiu-se necessidade de se realizar nova intervenção percutânea na lesão antes tratada ou encaminhamento do paciente para tratamento cirúrgico em decorrência de reestenose intrastent naquela lesão.

O seguimento foi realizado por meio de consulta clínica ou telefone em 30 dias, 6 meses, 1 ano e 2 anos após o procedimento ou sempre que necessário.

Esse estudo foi projetado de acordo com as Diretrizes e Normas Regulamentadoras de Pesquisas Envolvendo Seres Humanos (Resolução 196/1996 do Conselho Nacional de Saúde) e foi aprovado pelo Comitê de Ética em Pesquisa do hospital em que foi realizado. Todos os pacientes assinaram um termo de consentimento pós-informado.

\section{Intervenção coronária percutânea}

O implante dos stents nos pacientes do estudo foi realizado por via femoral, utilizando-se cateteres-guia $6 \mathrm{~F}$ ou $7 \mathrm{~F}$. Antes da angioplastia, eram administradas $10.000 \mathrm{UI}$ de heparina endovenosa e 0,5 mg de nitroglicerina intracoronariana. Todos os pacientes faziam uso prévio de AAS e ticlopidina ou clopidogrel iniciados três dias antes do procedimento. Caso o paciente não fizesse esse preparo, era administrada dose de ataque de $300 \mathrm{mg}$ de clopidogrel e $100 \mathrm{mg}$ de AAS antes do procedimento. Após o procedimento, os pacientes eram orientados a manter o AAS indefinidamente e o outro antiagregante por apenas 30 dias.

Sempre que possível, foi realizado implante direto do stent. Os stents eram escolhidos de modo a cobrir inteiramente a lesão e mais de um stent era permitido em caso de dissecção de borda, cobertura incompleta da lesão ou resultado subótimo. Os modelos de stent utilizados foram semelhantes, porém não totalmente idênticos. Apesar disso, não existem diferenças significativas na evolução clínica de pacientes tratados pelos tipos de stent utilizados nesse estudo na literatura. Foram eles: Helistent (Hexacath - Rueil-Malmaison, França), BX Sonic (Cordis - Miami, Estados Unidos), Liberté ou Express (Boston Scientific - Natick, Estados Unidos) e Driver (Medtronic - Minneapolis, Estados Unidos). Todos esses stents foram utilizados em porcentuais semelhantes no grupo dos stents convencionais.

Os critérios de sucesso adotados para os procedimentos foram: grau de estenose do vaso após a intervenção menor que 50\% e ausência de complicações maiores (morte ou infarto agudo do miocárdio) nas primeiras 24 horas após o procedimento.

\section{Angiografia coronariana quantitativa}

A angiografia coronariana quantitativa foi realizada off-line, tomando-se a projeção na qual se podia demonstrar a lesão mais grave, utilizando-se software com algoritmo de detecção automática de contorno (CAAS II, Pie Medical Imaging - Maastricht, Holanda), conforme descrito previamente ${ }^{16}$.

\section{Análise estatística}

Todas as variáveis foram testadas para normalidade pelo teste de Shapiro-Wilks. Na análise descritiva, as variáveis numéricas foram apresentadas como média \pm desvio padrão ou medianas e interquartis e as categóricas, como números (n) e porcentagens (\%). O teste de qui-quadrado e o teste exato de Fisher foram usados para variáveis categóricas; o teste $t$ de Student não-pareado e o teste de Mann-Whitney foram usados para variáveis contínuas. As características clínicas e angiográficas foram comparadas entre os grupos Titan e stent convencional. As curvas de sobrevida para ausência de eventos cardiovasculares adversos maiores e angina foram construídas pelo método de KaplanMeier e comparadas pelo teste de log-rank. A análise estatística foi realizada com os programas Stata SE 10 (Stata Corporation - Houston, Estados Unidos) e R 2.8.1. Valores de $\mathrm{P}<0,05$ foram considerados estatisticamente significantes, sendo todos os testes bicaudais.

\section{RESULTADOS}

Durante um período de 8 meses consecutivos, 243 pacientes foram admitidos no Serviço para se submeterem a angioplastia coronariana com implante de stent. Desses pacientes, 23 apresentaram infarto agudo do miocárdio, 5 apresentaram alergia ao AAS ou à ticlopidina, e 15 se recusaram a participar do protocolo do estudo, sem mencionar motivos específicos. Dessa forma, 200 pacientes foram incluídos no estudo.

As características clínicas desses pacientes podem ser analisadas na Tabela 1. A maioria dos pacientes apresentava angina estável (59,5\%) e doença multiarterial (uniarteriais: $32 \%$; biarteriais: $47,5 \%$; triarteriais: $20,5 \%$ ). Diabetes estava presente em $24 \%$ dos pacientes do grupo stent convencional e em $28 \%$ dos pacientes do grupo Titan $(P=0,63)$. Não houve diferença significativa entre as características clínicas nos dois grupos.

As características angiográficas das lesões podem ser observadas na Tabela 2. A maior parte das lesões $(58,3 \%)$ era do tipo B2/C. Não houve diferença entre os diâmetros de referência ou em relação ao comprimento da lesão entre os grupos stent convencional e Titan (15,5 mm vs. 15,3 mm; $\mathrm{P}=0,52)$.

Todos os procedimentos nos dois grupos foram bem-sucedidos. No grupo stent convencional foi implantado 1,2 stent por paciente e no grupo Titan, 1,1. 
TABELA 1

Características clínicas dos pacientes

\begin{tabular}{|c|c|c|c|c|}
\hline & \multirow{2}{*}{$\begin{array}{l}\text { Pacientes } \\
(\mathbf{n}=200)\end{array}$} & \multicolumn{2}{|c|}{ Grupos } & \multirow[b]{2}{*}{$\mathbf{P}$} \\
\hline & & Stent metálico $(n=100)$ & Titan $(n=100)$ & \\
\hline Idade, anos & $61,2 \pm 10,1$ & $60,6 \pm 10$ & $61,9 \pm 10,2$ & 0,34 \\
\hline Sexo feminino, \% & 35,5 & 32 & 39 & 0,37 \\
\hline Sintomas, n (\%) & & & & 0,841 \\
\hline Angina estável & $119(59,5)$ & $59(59)$ & $61(61)$ & \\
\hline Isquemia silenciosa & $44(22)$ & $24(24)$ & $20(20)$ & \\
\hline Angina instável & $37(18,5)$ & $17(17)$ & $20(20)$ & \\
\hline \multicolumn{5}{|l|}{ Fatores de risco, n (\%) } \\
\hline Hipertensão arterial & $158(79)$ & $84(84)$ & $74(74)$ & 0,12 \\
\hline Dislipidemia & $110(55)$ & $53(53)$ & $57(57)$ & 0,67 \\
\hline HF de DAC & $80(40)$ & $38(38)$ & $42(42)$ & 0,66 \\
\hline Tabagismo & $50(25)$ & $29(29)$ & $21(21)$ & 0,25 \\
\hline Diabetes & $52(26)$ & $24(24)$ & $28(28)$ & 0,63 \\
\hline IAM prévio, n (\%) & $101(50,5)$ & $51(51)$ & $50(50)$ & $>0,99$ \\
\hline ICP prévia, n (\%) & $16(8)$ & $4(4)$ & $12(12)$ & 0,06 \\
\hline RM prévia, n (\%) & $2(1)$ & $1(1)$ & $1(1)$ & $>0,99$ \\
\hline Fração de ejeção, \% & $59,2 \pm 14$ & $59,1 \pm 13$ & $59,2 \pm 14$ & 0,94 \\
\hline Doença multiarterial, n (\%) & $136(68)$ & $67(67)$ & $69(69)$ & 0,88 \\
\hline
\end{tabular}

Variáveis contínuas representadas por média \pm desvio padrão. HF de DAC = história familiar de doença arterial coronariana; IAM = infarto agudo do miocárdio; ICP = intervenção coronária percutânea; $\mathrm{n}=$ número de pacientes; RM = cirurgia de revascularização do miocárdio.

TABELA 2

Características angiográficas: análise por lesão

\begin{tabular}{|c|c|c|c|c|}
\hline & \multirow[b]{2}{*}{ Lesões ( $n=228)$} & \multicolumn{2}{|l|}{ Grupos } & \multirow[b]{2}{*}{$\mathbf{P}$} \\
\hline & & Stent convencional $(n=120)$ & Titan $(n=108)$ & \\
\hline \multicolumn{5}{|l|}{ Artéria coronária, n (\%) } \\
\hline Descendente anterior & $133(58,3)$ & $77(64,2)$ & $56(51,8)$ & 0,08 \\
\hline Circunflexa & $41(18)$ & $23(19,2)$ & $18(16,7)$ & 0,73 \\
\hline Coronária direita & $54(23,7)$ & $20(23,2)$ & $34(28,8)$ & 0,01 \\
\hline $\mathrm{ACC} / \mathrm{AHA} \mathrm{B} 2 / \mathrm{C}, \mathrm{n}(\%)$ & $133(58,3)$ & $70(58,3)$ & $63(58,3)$ & $>0,99$ \\
\hline Diâmetro de referência pré-ICP, mm & $2,81(2,4 ; 3,2)$ & $2,84(2,5 ; 3,2)$ & $2,76(2,4 ; 3,1)$ & 0,15 \\
\hline Diâmetro luminal mínimo pré-ICP, mm & $0,84(0,64 ; 1,04)$ & $0,85(0,61 ; 1,07)$ & $0,83(0,68 ; 1,04)$ & 0,71 \\
\hline Grau de estenose pré-ICP, \% & $70(63 ; 76)$ & $71(64 ; 78)$ & $70(62 ; 75)$ & 0,20 \\
\hline Comprimento da lesão, mm & $15,4(10,1 ; 20,6)$ & $15,5(10,6 ; 20,8)$ & $15,3(9,3 ; 20,1)$ & 0,52 \\
\hline Diâmetro de referência pós-ICP, mm & $3,1 \pm 0,49$ & $3,09 \pm 0,51$ & $3,13 \pm 0,48$ & 0,52 \\
\hline Diâmetro luminal mínimo pós-ICP, mm & $2,76 \pm 0,48$ & $2,75 \pm 0,42$ & $2,76 \pm 0,44$ & 0,83 \\
\hline Grau de estenose pós-ICP, \% & $12(8 ; 15)$ & $11,5(8 ; 15)$ & $12(9 ; 15)$ & 0,47 \\
\hline
\end{tabular}

Variáveis contínuas representadas por média \pm desvio padrão ou mediana (interquartis). ACC/AHA = American College of Cardiology/

American Heart Association; ICP = intervenção coronária percutânea; $\mathrm{n}=$ número de lesões.

\section{Seguimento clínico}

A Tabela 3 demonstra o seguimento clínico aos 30 dias e 2 anos. Ao analisar os eventos após 30 dias, verificou-se trombose subaguda no grupo Titan, que ocorreu 36 horas após o procedimento, tratada com sucesso por meio de angioplastia com balão. Os dois eventos no grupo stent convencional foram infarto agudo do miocárdio não-Q, caracterizados pelo aumento dos níveis de CK-MB maiores que 2,5 vezes o valor basal. Ambos os casos foram conduzidos clinicamente. 
Sant'Anna FM, et al. Estudo Randomizado e Comparativo da Intervenção Coronária Percutânea com Stents Recobertos por Titânio-Óxido Nítrico ou de Aço Inoxidável em Pacientes com Doença Arterial Coronária: Estudo RIO. Rev Bras Cardiol Invas. 2009;17(1):69-75.

TABELA 3

Seguimento clínico

\begin{tabular}{lcccc}
\hline & & \multicolumn{2}{c}{ Grupos } & \\
\cline { 2 - 4 } & Pacientes & Stent metálico & Titan & P \\
\hline ECAM 1 mês, $n$ (\%) & & & 100 & \\
Número de pacientes & 200 & 100 & $1(1)$ & 0,56 \\
Morte/IAM/RLA & $3(1,5)$ & $2(2)$ & 0 & NA \\
Morte & 0 & 0 & $1(1)$ & 0,56 \\
IAM & $3(1,5)$ & $2(2)$ & $1(1)$ & $>0,99$ \\
RLA & $1(0,5)$ & 0 & $1(1)$ & $>0,99$ \\
Trombose subaguda & $1(0,5)$ & 0 & 63 & \\
ECAM 2 anos, $n$ (\%) & & & $4(6,4)$ & 0,036 \\
Número de pacientes & 131 & $12(17,6)$ & $2(3,2)$ & 0,02 \\
Morte/IAM/RLA & $16(12,2)$ & $11(16,2)$ & $2(3,2)$ & 0,61 \\
IAM/RLA & $13(10)$ & $1(1,5)$ & $1(1,6)$ & 0,12 \\
Morte & $3(2,3)$ & $6(8,8)$ & $2(3,2)$ & 0,44 \\
IAM & $7(5,3)$ & $5(7,4)$ & $10(15,9)$ & 0,02 \\
RLA & $7(5,3)$ & $20(29,4)$ & \\
Angina (2 anos), $n(\%)$ & $30(23)$ & &
\end{tabular}

ECAM = eventos cardíacos adversos maiores; IAM = infarto agudo do miocárdio; $\mathrm{n}$ = número de pacientes; RLA = revascularização da lesão-alvo.

Após 2 anos pode-se observar incidência maior de eventos cardiovasculares adversos maiores no grupo stent convencional, quando comparado ao grupo Titan, principalmente quanto aos eventos combinados infarto agudo do miocárdio/nova revascularização da lesão-alvo $(16,2 \%$ vs. 3,2\%; P = 0,02). A Figura 1 mostra a curva de sobrevida livre de eventos cardíacos nos dois grupos, significativamente melhor no grupo Titan quando comparado ao grupo stent convencional $(93,6 \%$ vs. $82,4 \%$; $P=0,036)$. Em relação à presença de angina, também podemos observar vantagem do grupo Titan (15,9\% vs. 29,4\%; $\mathrm{P}=0,02)$.

Ambos os óbitos que ocorreram no grupo Titan foram decorrentes de causas não-cardíacas. Como os pacientes desse estudo eram oriundos de diferentes cidades, houve perda de $34,5 \%$ dos pacientes no seguimento de 2 anos. A perda no grupo Titan foi de $37 \%$ e de $32 \%$ no grupo stent convencional $(P=0,55)$.

\section{DISCUSSÃO}

Os resultados desse estudo confirmam que a utilização do stent Titan no tratamento percutâneo da insuficiência coronariana crônica confere vantagens em relação ao stent convencional de aço inoxidável, cursando com taxas de sobrevida livre de eventos cardiovasculares adversos maiores e angina significativamente maiores. O estudo RIO é, até onde se sabe, o maior estudo clínico randomizado em insuficiência coronariana crônica envolvendo a utilização do stent recoberto por titânio-óxido nítrico até o momento, com seguimento de 2 anos.

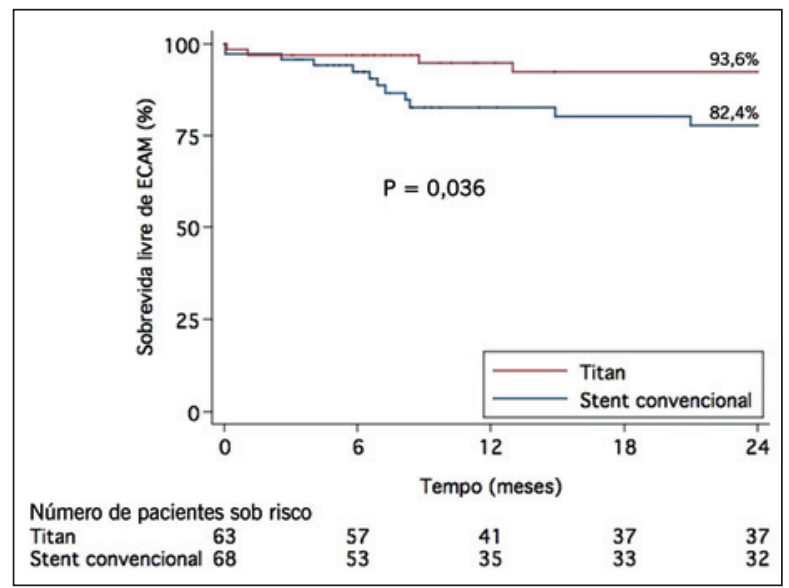

Figura 1 - Curva de sobrevida livre de eventos cardíacos adversos maiores (ECAM) após dois anos.

A primeira característica importante desse estudo foram os baixos índices de trombose subaguda do stent com base nos critérios da Academic Research Consortium $(\mathrm{ARC})^{17}$, apesar de todos os pacientes em ambos os grupos terem feito uso apenas de ticlopidina ou clopidogrel durante 30 dias associados ao AAS indefinidamente. Alguns estudos demonstraram que os stents recobertos por titânio-óxido nítrico parecem ter superfície menos trombogênica que os stents convencionais ou mesmo que os stents eluidores de fármacos ${ }^{13-15}$. Outro dado importante é o impacto econômico que isso produz, pois pacientes que se submetem ao implante de stents farmacológicos, além do 
Sant'Anna FM, et al. Estudo Randomizado e Comparativo da Intervenção Coronária Percutânea com Stents Recobertos por Titânio-Óxido Nítrico ou de Aço Inoxidável em Pacientes com Doença Arterial Coronária: Estudo RIO. Rev Bras Cardiol Invas. 2009;17(1):69-75.

elevado custo da endoprótese, têm também que fazer uso de clopidogrel por no mínimo um ano, o que representa aumento ainda maior do custo total do procedimento $^{18}$.

O único estudo clínico randomizado na insuficiência coronariana crônica comparando o stent Titan com o stent de aço inoxidável foi publicado por Windecker et al. ${ }^{12}$ (TiNOX Trial). Esse estudo, elaborado com o intuito de investigar a segurança e a eficácia do Titan comparado ao stent convencional, demonstrou baixos índices de eventos cardiovasculares adversos maiores no grupo Titan, em comparação com o grupo stent convencional ( $7 \%$ vs. $27 \%$; $P=0,02)$, principalmente pela necessidade de nova revascularização da lesão-alvo ( $7 \%$ vs. $23 \%$; $P=0,07)$. Todos os pacientes desse estudo foram submetidos a controle angiográfico, o que talvez explique os índices relativamente altos de nova intervenção nos pacientes do grupo stent convencional, bem maiores que os encontrados em nosso estudo. De qualquer maneira, podemos observar nesse estudo taxas de eventos cardiovasculares adversos maiores no grupo Titan semelhantes às encontradas em nossa casuística $(7 \%$ vs. $6,4 \%$ ), o que corrobora o bom desempenho clínico do stent Titan. Nosso estudo, por contar com mais pacientes e tempo maior de seguimento, foi mais apropriado para analisar os desfechos clínicos que o estudo de Windecker et al. ${ }^{12}$, cujo objetivo primário era analisar a segurança e a eficácia do stent Titan.

Foram publicados, até o momento, três registros clínicos sobre a utilização do stent Titan em doença arterial coronariana no mundo real, dois registros multicêntricos descrevendo a experiência com a utilização do Titan na prática diária (Titan-PORI e Titanium$\mathrm{NO})^{13,14}$, e um terceiro registro prospectivo (TITANOX) comparando o stent Titan com o stent eluidor de paclitaxel ${ }^{15}$. É interessante observar que todos esses registros incluíram pacientes com infarto agudo do miocárdio, que responderam por pelo menos um terço da população desses estudos. O estudo Titan-PORI ${ }^{13}$ investigou a incidência de eventos cardiovasculares adversos maiores 9 meses após implante do stent Titan em 193 pacientes (210 lesões). A taxa de eventos cardiovasculares adversos maiores desse registro foi de 10,4\% (4,1\%, infarto agudo do miocárdio; 0\%, óbito; e 8,3\%, revascularização da lesão-alvo). O estudo Titanium- $\mathrm{NO}^{14}$ analisou a incidência de eventos cardiovasculares adversos maiores 6 meses após implante do Titan em 296 pacientes (333 lesões) e seus achados foram semelhantes aos do Titan-PORI $(7,6 \%$ de eventos cardiovasculares adversos maiores: 0,7\%, infarto agudo do miocárdio; 0,7\%, óbito; e 5,4\%, revascularização da lesão-alvo). Já o registro TITANOX comparou 201 pacientes (218 lesões) que receberam o Titan com 204 pacientes (224 lesões) que receberam o stent eluidor de paclitaxel no mesmo período, analisando seus índices de eventos cardiovasculares adversos maiores após 30 dias e 1 ano. As taxas de eventos cardiovasculares adversos maiores após 1 ano foram semelhantes nos dois grupos $(10,9 \%$ Titan vs. $13,7 \%$ stent eluidor de paclitaxel; $P=0,40$ ), sendo as taxas de revascularização da lesão-alvo nos dois grupos de $5 \%$ e $4,9 \%$, respectivamente. Chama a atenção nesse estudo a diferença dos índices de infarto agudo do miocárdio entre os dois grupos $(4,5 \%$ Titan vs. $10,3 \%$ stent eluidor de paclitaxel; $P=0,025)$, decorrente do alto índice de trombose subaguda do stent no grupo stent eluidor de paclitaxel $(3,4 \%)$ contra $0 \%$ no grupo Titan. Talvez isso se deva ao fato de grande parte da população do estudo ter sido de pacientes cuja indicação de intervenção percutânea foi infarto agudo do miocárdio. Outro dado que merece ser destacado foram os baixos índices de eventos cardiovasculares adversos maiores em todos esses registros, principalmente se levarmos em conta a complexidade clínica dos pacientes tratados. Nosso estudo demonstrou taxas de eventos cardiovasculares adversos maiores bastante semelhantes a esses registros clínicos, o que o aproxima adequadamente do chamado "mundo real".

\section{Limitações}

Existem algumas limitações no presente estudo. Em primeiro lugar, o alto índice de perda de pacientes no seguimento tardio. Infelizmente, a maioria de nossos pacientes provinha de diferentes cidades, algumas muito distantes. Isso, aliado ao fato de serem também pacientes carentes e com poucas condições financeiras para ficar se locomovendo de um local a outro, levou a essa perda relativamente alta. Em segundo lugar, esse estudo não foi duplo cego. O médico sabia qual stent estava sendo implantado em determinado paciente. Apesar disso, como os eventos foram analisados ao longo de 2 anos e os pacientes foram sorteados, não cremos que esse fato tenha alterado nossos resultados. Em terceiro lugar, o desenho de nosso estudo não incluiu controle angiográfico ou acompanhamento de rotina por testes não-invasivos. Assim, certamente as taxas de isquemia silenciosa ou reestenose angiográfica foram subestimadas. Por outro lado, basear nosso seguimento em critérios clínicos certamente evitou revascularizações da lesão-alvo desnecessárias decorrentes do reflexo "óculo-estenótico" ou mesmo à ansiedade extrema dos pacientes nesses casos. Em quarto lugar, o tamanho de nossa amostra foi relativamente pequeno, principalmente em decorrência de perda tardia. Ainda assim, o total dos pacientes seguidos ao longo de 2 anos (131) foi superior ao número necessário para que nossos resultados fossem válidos estatisticamente (128 pacientes, 64 em cada grupo). De qualquer maneira, é importante que um estudo maior e mais robusto seja conduzido, de modo a confirmar esses resultados. Em quinto lugar, o grupo dos stents convencionais utilizou três stents de empresas diferentes, todos de aço inoxidável, porém com desenhos ligeiramente diversos. Todavia, os resultados da literatura referentes à utilização desses stents 
Sant'Anna FM, et al. Estudo Randomizado e Comparativo da Intervenção Coronária Percutânea com Stents Recobertos por Titânio-Óxido Nítrico ou de Aço Inoxidável em Pacientes com Doença Arterial Coronária: Estudo RIO. Rev Bras Cardiol Invas. 2009;17(1):69-75.

mostram-se semelhantes em termos de eventos clínicos precoces e tardios. E, em sexto e último lugar, comparamos o stent Titan com o stent convencional de aço inoxidável e não com o stent farmacológico, que hoje é o padrão de referência no tratamento percutâneo da doença aterosclerótica coronariana. No entanto, a utilização do stent convencional ainda é a rotina na maior parte dos centros de cardiologia em nosso País, por ser esse o único stent coberto pelo Sistema Único de Saúde. Buscamos com isso realizar um estudo que refletisse adequadamente nossa prática diária.

\section{CONCLUSÃO}

Esse estudo demonstrou que a utilização do stent recoberto por titânio-óxido nítrico no tratamento percutâneo de pacientes com insuficiência coronariana crônica cursa com baixos índices de eventos cardiovasculares adversos maiores e angina após 2 anos, apresentando resultados melhores que os obtidos com o stent de aço inoxidável.

\section{CONFLITO DE INTERESSES}

Os autores declararam inexistência de conflito de interesses.

\section{REFERÊNCIAS BIBLIOGRÁFICAS}

1. Peterson ED, Cowper PA, DeLong ER, Zidar JP, Stack RS, Mark DB. Acute and long-term cost implications of coronary stenting. J Am Coll Cardiol. 1999;33(6):1610-8.

2. Al Suwaidi J, Berger PB, Holmes DR Jr. Coronary artery stents. JAMA. 2000;284(14):1828-36.

3. Kastrati A, Hall D, Schömig A. Long-term outcome after coronary stenting. Curr Control Trials Cardiovasc Med. 2000; $1(1): 48-54$

4. Moses JW, Leon MB, Popma JJ, Fitzgerald PJ, Holmes DR, O'Shaughnessy C, et al.; SIRIUS Investigators. Sirolimus-eluting stents versus standard stents in patients with stenosis in a native coronary artery. N Engl J Med. 2003;349(14):1315-23.

5. Garasic JM, Edelman ER, Squire JC, Seifert P, Williams MS, Rogers C. Stent and artery geometry determine intimal thickening independent of arterial injury. Circulation. 2000;101(7): 812-8.
6. Kastrati A, Mehilli J, Dirschinger J, Dotzer F, Schühlen $H$, Neumann FJ, et al. Intracoronary stenting and angiographic results: strut thickness effect on restenosis outcome (ISARSTEREO) trial. Circulation. 2001;103(23):2816-21.

7. Babapulle MN, Eisenberg MJ. Coated stents for the prevention of restenosis. Part II. Circulation. 2002;106(22):2859-66.

8. Köster R, Vieluf D, Kiehn M, Sommerauer M, Kähler J, Baldus S, et al. Nickel and molybdenum contact allergies in patients with coronary in-stent restenosis. Lancet. 2000; 356(9245):1895-7.

9. Steinemann SG. Titanium: the material of choice? Periodontol 2000. 1998;17:7-21.

10. Steinemann SG. Metal implants and surface reactions. Injury. 1996;27(Suppl 3):SC16-22.

11. Windecker S, Mayer I, De Pasquale G, Maier W, Dirsch O, De Groot P, et al; Working Group on Novel Surface Coating of Biomedical Devices (SCOL). Stent coating with titaniumnitride-oxide for reduction of neointimal hyperplasia. Circulation. 2001;104(8):928-33.

12. Windecker $S$, Simon R, Lins $M$, Klauss V, Eberli FR, Roffi $M$, et al. Randomized comparison of a titanium-nitride-oxidecoated stent with a stainless steel stent for coronary revascularization: the TINOX trial. Circulation. 2005;111(20): 2617-22.

13. Karjalainen PP, Ylitalo A, Airaksinen JK. Real world experience with the TITAN stent: a 9-month follow-up report from The Titan PORI Registry. Eurolntervention. 2006;2:187-91.

14. Mosseri M, Miller H, Tamari I, Plich M, Hasin Y, Brizines M. The titanium-NO stent: results of a multicenter registry. Eurolntervention. 2006;2:192-6.

15. Karjalainen PP, Ylitalo A, Airaksinen JK. Titanium and nitride oxide-coated stents and paclitaxel-eluting stents for coronary revascularization in an unselected population. J Invasive Cardiol. 2006;18(10):462-8.

16. Reiber JH, Serruys PW, Kooijman CJ, Wijns W, Slager CJ, Gerbrands JJ, et al. Assessment of short-, medium-, and longterm variations in arterial dimensions from computer-assisted quantitation of coronary cineangiograms. Circulation. 1985;71(2):280-8.

17. Applegate RJ, Sacrinty MT, Little WC, Santos RM, Gandhi SK, Kutcher MA. Incidence of coronary stent thrombosis based on academic research consortium definitions. Am J Cardiol. 2008;102(6):683-8.

18. Cowper PA, Udayakumar K, Sketch MH Jr, Peterson ED. Economic effects of prolonged clopidogrel therapy after percutaneous coronary intervention. J Am Coll Cardiol. 2005; 45(3):369-76. 